\section{Antimicrobial activity of four essential oils against pigmenting Pseudomonas fluorescens and biofilm- producing Staphylococcus aureus of dairy origin}

Francesca Pedonese, ${ }^{1}$ Filippo Fratini, Luisa Pistelli, ${ }^{2}$ Federica Maria Porta, ${ }^{1}$ Pierluigi Di Ciccio, ${ }^{3}$ Roberto Fischetti, ${ }^{4}$ Barbara Turchi, ${ }^{1}$ Roberta Nuvoloni ${ }^{1}$

${ }^{1}$ Department of Veterinary Sciences, University of Pisa, Pisa; ${ }^{2}$ Department of Pharmacy, University of Pisa, Pisa; ${ }^{3}$ Food and Drug Department, University of Parma, Parma; ${ }^{4}$ Institute for Experimental Veterinary Medicine of Lazio and Tuscany, Pisa, Italy

\begin{abstract}
Essential oils (EOs) are mixtures of secondary metabolites of plant origin with many useful properties, among which the antimicrobial activity is also of interest for the food industry. EOs can exert their antimicrobial potential both directly, in food products and active packaging, and indirectly, as sanitizing and anti-biofilm agents of food facility surfaces. Aim of this research was to evaluate the antimicrobial activity of four EOs (bergamot, cinnamon, manuka and thyme) against Pseudomonas fluorescens and Staphylococcus aureus isolated from milk and dairy products. The chemical composition of EOs was evaluated by Gas Chromatography-Mass Spectrometry analysis. Minimum Inhibitory Concentration values were determined by a microplate method against 9 Ps. fluorescens from marketed mozzarella with blue discoloration defect, and 3 biofilm-producing $S$. aureus from milk. Reference ATCC strains were included. Pigment production activity by Ps. fluorescens was assessed both in culture and in cheese. EOs of manuka (leptospermone $23 \%$ ) and thyme (carvacrol 30\%, pcymene $20 \%$, thymol $15 \%$ ) showed the highest antimicrobial activity against $S$. aureus, MIC values were $0.012 \%-0.024 \%$ and $0.024 \% \mathrm{v} / \mathrm{v}$, respectively; meanwhile EOs from thyme and cinnamon (cinnamaldehyde $55 \%$ ) exhibited the best activity against $P$ s. fluorescens with MIC values of $0.098 \%-0.195 \%$ and $0.195 \%-0.391 \%$ $\mathrm{v} / \mathrm{v}$, respectively. The antimicrobial activity of these EOs is promising and they could be exploited in the dairy production chain.
\end{abstract}

\section{Introduction}

Essential oils (EOs) are aromatic and volatile mixtures of secondary metabolites obtained from different parts of plants. EOs are composed of many bioactive molecules, mainly terpenes, terpenoids and phenylpropenes, and their actual antimicrobial effect depends on many intrinsic and environmental factors (Hyldgaard et al., 2012). Their use in food industry is aimed to provide flavours and to improve safety and quality of products. They can be employed directly in food, or as components of food packaging (Lucera et al., 2012) and even in sanification of food processing environments and food-contact surfaces due to their antimicrobial and anti-biofilm potential (Valeriano et al., 2012).

The present work was aimed to evaluate the antimicrobial activities of four EOs (Citrus bergamia Risso, Cinnamomum zeylanicum L., Leptospermum scoparium J.R. et G. Forst and Thymus vulgaris L.) on food-related bacteria: Pseudomonas fluorescens, isolated from mozzarella cheese with blue discoloration, and biofilm-producing Staphylococcus aureus from milk. Gas chromatography-mass spectrometry (GC-MS) analyses were carried out to perform the chemical characterization of the four tested EOs.

\section{Materials and Methods}

\section{Bacterial cultures}

A total of 10 Ps. fluorescens was used in this study: 9 wild isolates (PS59, PS60, PS63, PS70, PS71, PS231, PS249, PS251 and PS282) from commercial samples of spoiled mozzarella cheeses with blue pigmentation and the reference strain PS. fluorescens ATCC 13525. The wild isolates, provided by Istituto Zooprofilattico Sperimentale Lazio-Toscana (Pisa), were phenotypically identified by API 20 NE (bioMérieux, Marcy-l'Etoile, France). Moreover, the identification was confirmed genetically according to Scarpellini et al. (2004). After DNA extraction by boiling at $95^{\circ} \mathrm{C}$ for $10 \mathrm{~min}$, the species-specific PCR was performed using the KAPA Taq ReadyMix PCR kit (Kapa Biosystems, Boston, MA, USA).

The pigmentation capacity of pseudomonads was studied in mozzarella cheese and in a lab-made minimal nutritive broth used for the cultivation and maintenance of Pseudomonas fluorescens and other microorganisms (Yeast Extract Glucose Broth, YEGB, https://www.bioworld.com/productinfo/3_43_287_688/101
Correspondence: Francesca Pedonese, Department of Veterinary Sciences, University of Pisa, Viale delle Piagge 2, 56124 Pisa, Italy.

Tel: +39-050-2216707 - Fax: +39-050-2210654.

E-mail: francesca.pedonese@unipi.it

Key words: Essential oils, Gas chromatography-mass spectrometry, Pseudomonas fluorescens, Staphylococcus aureus, Dairy.

Conflict of interest: the authors declare no potential conflict of interest.

Funding: this research was funded by Pisa University grants.

Received for publication: 21 July 2017. Revision received: 11 September 2017. Accepted for publication: 12 September 2017.

This work is licensed under a Creative Commons Attribution-NonCommercial 4.0 International License (CC BY-NC 4.0).

(C) Copyright F. Pedonese et al., 2017

Licensee PAGEPress, Italy

Italian Journal of Food Safety 2017; 6:6939

doi:10.4081/ijfs.2017.6939

74/Yeast-Extract-Glucose-Broth.html). YEGB had the following composition: glucose $1.5 \mathrm{~g} / \mathrm{L}$, di-potassium hydrogen orthophosphate $5.2 \mathrm{~g} / \mathrm{L}$, potassium dihydrogen phosphate $3.18 \mathrm{~g} / \mathrm{L}$, magnesium sulfate $0.12 \mathrm{~g} / \mathrm{L}$, yeast extract $0.5 \mathrm{~g} / \mathrm{L}$, ammonium chloride $0.54 \mathrm{~g} / \mathrm{L}$. Marketed citric mozzarella samples were inoculated with a final count of about $10^{2} \mathrm{CFU} / \mathrm{mL}$ in preservation liquid. Samples were stored at $10^{\circ} \mathrm{C}$ for 72 hours in their packaging and then opened, maintained at the same temperature and observed daily up to 10 days. Pseudomonas quantitative determination on Pseudomonas Agar with CFC Supplement (Oxoid, Basingstoke, UK) was carried out to exclude the presence of pseudomonads in the preservation liquid. As for YEGB assay, each isolate wasgrown overnight at $25^{\circ} \mathrm{C}$ in YEGB, and ten-fold serial dilutions in sterile saline solution were prepared. Ten $\mu \mathrm{L}$ of the dilutions were seeded, in 5 different Eppendorf tubes per isolate, to obtain final counts of pseudomonads ranging from $10^{1}$ to $10^{5} \mathrm{CFU} / \mathrm{mL}$. Each tube, of $1.5 \mathrm{~mL}$ declared capacity, was filled to the top with $1.8 \mathrm{~mL}$ of YEGB to maximize the surface of broth culture exposed to air. Eppendorf tubes were left open, incubated at $25^{\circ} \mathrm{C}$ and observed daily up to 7 days.

Among biofilm-producing S. aureus, 3 strains, isolated from raw milk, came from the collection of Food and Drug Department, Parma (PA1 and PA2) and from that of Veterinary Science 
Department, Pisa (PI1) and their biofilmproducing capacity had been previously assessed following Di Ciccio et al. (2015), the other 2 were reference strains, $S$. aureus ATCC 35556 (strong producer) and $S$. aureus ATCC 12600 (intermediate producer). S. aureus PA1 and $S$. aureus PA2 had been previously characterized as methicillin-resistant (MRSA) (Di Ciccio et al., 2016); S. aureus PI1 was a methicillin-susceptible, enterotoxin $\mathrm{C}$ producer (Pedonese et al., 2014).

\section{Essential oils}

EOs (from Flora s.r.l., Lorenzana, Pisa, Italy) of bergamot (C. bergamia Risso, $\mathrm{Cb}$ ), cinnamon bark (C. zeylanicum L., in $40 \%$ ethanol, Cz), manuka leaves (L. scoparium J. R. et G. Forst, Ls) and thymus (T. vulgaris L., Tv) were used.

\section{Gas chromatography-mass spec- trometry analysis}

The chemical composition of EOs was determined by using Gas ChromatographyElectron Impact Mass Spectrometry (GCMS). Analyses were performed with a Varian CP-3800 gas chromatograph equipped with a DB-5 capillary column (30 $\mathrm{m} \times 0.25 \mathrm{~mm}$; coating thickness $0.25 \mu \mathrm{m}$ ) and a Varian Saturn 2000 ion trap mass detector. Analytical conditions were those reported by Fratini et al. (2014). The identification of the constituents was based on comparison of retention times with those of pure authentic samples, comparing their retention indices relative (LRI) to the series of $n$-hydrocarbons, by computer matching against commercial libraries (NIST 98 and ADAMS) (Adams, 1995) and homemade library mass spectra, built up from pure substances, known oils and MS literature data (Stenhagen et al., 1974).

\section{Minimal inhibitory concentration determination assay}

MIC values of EOs, i.e. the lowest concentration that inhibits the visible microbial growth, were determined following Wiegand et al. (2008) with minor modifications. The assay was performed in sterile 96-well microtiter plates using $10 \mu \mathrm{L}$ of bacterial inoculum and $190 \mu \mathrm{L}$ of each EOs dilution. EOs dilutions were prepared in Tryptone Soy Broth (Oxoid) supplemented with dimethyl sulfoxide (DMSO) to a final ratio of 1:3:4 (v/v) and a two-fold dilution series was performed from $1 / 8$ to $1 / 16384$. For the bacterial inoculum an overnight broth culture of each microorganism, spectrophotometrically (Ultrospec 2100 Pro, Amersham Biosciences, Buckinghamshire, GB) adjusted at $550 \mathrm{~nm}$ at about $1.5 \times 10^{8}$ $\mathrm{CFU} / \mathrm{mL}$, was used. Positive and negative controls, and for $\mathrm{Cz}$ also ethanol control,
Table 1. Chemical composition of essential oils detected by gas chromatography-mass spectrometry analysis.

\begin{tabular}{|c|c|c|c|c|c|c|}
\hline Compound & Class & LRI & $\mathrm{Cb}$ & $\mathrm{Cz}$ & Ls & Tv \\
\hline$\alpha$-pinene & $\mathrm{mh}$ & 940 & 1.1 & & 1.7 & 1.1 \\
\hline Sabinene & $\mathrm{mh}$ & 978 & 1.1 & & & \\
\hline$\beta$-pinene & $\mathrm{mh}$ & 981 & 6 & & & \\
\hline Myrcene & $\mathrm{mh}$ & 993 & & & & 1.3 \\
\hline$\alpha$-phellandrene & $\mathrm{mh}$ & 1006 & & 1.1 & & \\
\hline$\alpha$-terpinene & $\mathrm{mh}$ & 1019 & & & & 1.5 \\
\hline p-cymene & $\mathrm{mh}$ & 1026 & & & & 20.2 \\
\hline o-cymene & $\mathrm{mh}$ & 1026 & & 2.5 & & \\
\hline Limonene & $\mathrm{mh}$ & 1032 & 30.8 & & & \\
\hline$\beta$-phellandrene & $\mathrm{mh}$ & 1033 & & 4.4 & & \\
\hline$\gamma$-terpinene & $\mathrm{mh}$ & 1062 & 7.4 & & & 8.3 \\
\hline Linalool & om & 1102 & 12.5 & 5 & & 7.4 \\
\hline Camphor & om & 1148 & & & & 1 \\
\hline 4-terpineol & om & 1180 & & & & 1.8 \\
\hline (Z)-cinnamaldehyde & nt & 1223 & & 1.2 & & \\
\hline Linalool acetate & om & 1260 & 34.9 & & & \\
\hline (E)-cinnamaldehyde & nt & 1274 & & 55 & & \\
\hline Thymol & om & 1290 & 8 & & & 15.3 \\
\hline Carvacrol & om & 1301 & $>$ & & & 29.7 \\
\hline$\alpha$-cubebene & sh & 1351 & & & 3.2 & \\
\hline Eugenol & pp & 1361 & & 4.6 & & \\
\hline$\alpha$-copaene & sh & 1376 & & 1.6 & 4.8 & \\
\hline$\alpha$-gurjunene & sh & 1410 & & & 1 & \\
\hline$\beta$-caryophyllene & sh & 1418 & & 10.2 & 2.4 & 4.8 \\
\hline Aromadendrene & sh & 1441 & & & 1.8 & \\
\hline (E)-cinnamyl acetate & nt & 1449 & & 1.1 & & \\
\hline cis-muurola-3,5-diene & sh & 1450 & & & 5 & \\
\hline$\alpha$-humulene & sh & 1456 & & 2.8 & & \\
\hline trans-cadina-1(6),4-diene & sh & 1470 & & & 3.7 & \\
\hline Y-muurolene & sh & 1477 & & & 1.1 & \\
\hline$\beta$-selinene & sh & 1485 & & & 4.8 & \\
\hline trans-muurola-4-(14),5-diene & sh & 1494 & & & 1 & \\
\hline Viridiflorene & sh & 1495 & & & 5.3 & \\
\hline trans-calamenene & sh & 1522 & & & 15.2 & \\
\hline$\delta$-cadinene & sh & 1523 & & & 4.5 & \\
\hline$\alpha$-calacorene & sh & 1542 & & & 6.3 & \\
\hline Caryophyllene oxide & os & 1582 & & 1.4 & & \\
\hline iso-leptospermone & os & 1623 & & & 5.6 & \\
\hline Leptospermone & os & 1631 & & & 22.9 & \\
\hline Cubenol & os & 1647 & & & 1.1 & \\
\hline Benzylbenzoate & nt & 1766 & & 1.8 & & \\
\hline Unknown & & & 0.3 & 3.2 & 0 & 0.1 \\
\hline Mh & & & 48.4 & 10.3 & 2.7 & 34.4 \\
\hline Om & & & 49.2 & 6.2 & 0.2 & 58.1 \\
\hline Sh & & & 1.8 & 14.6 & 64.9 & 6.5 \\
\hline Os & & & 0 & 1.6 & 31.7 & 0.4 \\
\hline $\mathrm{Nt}$ & & & 0.3 & 59.5 & 0.5 & 0.5 \\
\hline Pp & & & 0 & 4.6 & 0 & 0 \\
\hline Total (unknown excluded) & & & 99.7 & 96.8 & 100.0 & 99.9 \\
\hline
\end{tabular}

LRI, linear retenction index; Cb, Citrus bergamia; Cz, Cinnamomum zeylanicum; Ls, Leptospermum scoparium; Tv, Thymus vulgaris; mh, monoterpene hydrocarbons; om, oxygenated monoterpenes; sh, sesquiterpenes hydrocarbons; os, oxygenated sesquiterpenes; nt, no terpene derivatives; pp, phenilpropanoids. Other compounds were detected at $<1 \%$. They were: for $\mathrm{Cb}, \alpha$-thujene, myrcene, p-cymene, (E)- $\beta$ ocimene, terpinolene, $\alpha$-terpineol, 1-octanyl acetate, neral, geranial, $\alpha$-terpinyl acetate, neryl acetate, geranyl acetate, trans- $\alpha$-bergamotene, (E)- $\beta$-farnesene, $\beta$-bisabolene, bergaptene; for $\mathrm{Cz}, \alpha$-thujene, $\alpha$-pinene, camphene, benzaldehyde, $\beta$-pinene, $\alpha$-terpinene, $\mathrm{p}$-mentha-2,4(8)diene, 4-terpineol, $\alpha$-terpineol, (Z)-ocimenone, humulene epoxide-II, tetradecanal; for Ls, $\beta$-pinene, myrcene, $p$-cymene, limonene, 1,8 -cineole, $\gamma$-terpinene, isopentylisovalerate, methylbutanoate, cyclosativene, $\beta$-elemene, $\beta$-copaene, $\alpha$-neoclovene, alloaromadendrene, $\alpha$-amorphene, $\alpha$-muurolene, $\alpha$-bulnesene, (E,E)- $\alpha$-farnesene, $\delta$-amorphene, trans- $\gamma$-cadinene, cadina-1,4-diene (cubenene), flavesone, (E) nerolidol, spathulenol, caryophyllene oxide, globulol, viridiflorol, valerianol; for Tv, $\alpha$-thujene, camphene, $\beta$-pinene, 1-octen-3-ol, 3-octanone, $\alpha$-phellandrene, cis-sabinene hydrate, terpinolene, borneol, p-cymen-8-ol, $\alpha$-terpineol, cis-dihydrocarvone, verbenone, methylcarvacrol, linalool acetate, isobornyl acetate, carvacrol acetate, $\alpha$-humulene, (E)- $\beta$-farnesene, $\gamma$-muurolene, viridiflorene, trans- $\gamma$-cadinene, $\delta$ cadinene, caryophyllene oxide. All these compounds were considered for calculating the total percentage of each class of constituents. 
were included. The microplates were incubated at $25^{\circ} \mathrm{C}$ (pseudomonads) and $37^{\circ} \mathrm{C}$ (staphylococci) for 24 hours avoiding evaporation. The assay was made in triplicate and the mode was determined.

\section{Results}

\section{Confirmation of identification and pigment production by Pseudomonas fluorescens}

All isolates were phenotypically identified as Ps. fluorescens with an API identification percentage range of $99.7 \%-99.9 \%$ and a $\mathrm{T}$ index range of $0.87-0.97$. Speciesspecific PCR results confirmed that all the isolates belonged to the species Ps. fluorescens. They were able to produce blue pigment in mozzarella cheese and in its preservation liquid, beginning from 2 days after the package opening, and more abundantly at the end of the trial. The pigmentation production was evident for all isolates except for PS63 and PS282. The lack of pigmentation was confirmed for these 2 isolates also in YEGB. All the other isolates showed a visible pigmentation at all dilutions with YEGB method beginning from 48 hours of incubation. The isolation of nopigment producing Ps. fluorescens from blue mozzarella cheeses indicates that cheese samples were contaminated with different strains.

\section{Essential oils composition}

GC-MS profile revealed the presence of $7,13,18$ and 11 different compounds with a percentage higher than $1 \%$ for $\mathrm{Cb}, \mathrm{Cz}$, Ls and $\mathrm{Tv}$, respectively (Table 1). The most representative compounds mainly included in monoterpenes and sesquiterpenes were linalool acetate $(34.9 \%)$ and limonene (30.8\%) for $\mathrm{Cb}$, leptospermone $(22.9 \%)$ and trans-calamenene $(15.2 \%)$ for Ls and carvacrol $(29.7 \%)$, p-cymene $(20.2 \%)$ and thymol (15.3\%) for Tv. Cz showed the non-terpene derivative (E)-cinnamaldehyde $(55 \%)$ together with the sesquiterpene $\beta$ caryophyllene $(10.2 \%)$, as main compounds.

\section{Determination of minimal inhibitory concentration against Pseudomonas fluorescens and Staphylococcus aureus}

Overall, the tested biofilm-producing $S$. aureus showed to be more sensitive than $P S$. fluorescens. Particularly, as shown in Table 2 , very promising MIC values were obtained for Ls $(0.012 \% \mathrm{v} / \mathrm{v}$ for 4 strains out of 5, among which the MRSA, and $0.024 \% \mathrm{v} / \mathrm{v}$ for the remaining one), which resulted even better than Tv $(0.024 \% \mathrm{v} / \mathrm{v})$. $\mathrm{Cz}$ evidenced an intermediate effect (0.049\%-0.098\% v/v), while $\mathrm{Cb}$ was poorly effective ( $\geq 0.781 \% \mathrm{v} / \mathrm{v})$. Ps. fluorescens growth was not affected by $\mathrm{Cb}$ and Ls, which were active only at high concentrations. Also Tv and $\mathrm{Cz}$ showed higher MIC values than those obtained for $S$. aureus (0.098\%-0.195\% and 0.195\%-0.391\% v/v, respectively). Ethanol solvent had negligible effect on the antimicrobial activity of $\mathrm{Cz}$ EO (MIC: $3.125 \% \mathrm{v} / \mathrm{v}$ ).

\section{Discussion}

The tested microorganisms, belonging to a pathogenic, $S$. aureus, and a spoilage species, Ps. fluorescens, have been chosen for their relevance for dairy industry. $S$. aureus is one of the most frequent pathogens associated with milk and dairy outbreaks (De Buyser et al., 2001). In recent years biofilm-producing and methicillin-resistant $S$. aureus, such as the wild ones tested in this study, have been recovered from food of animal origin, in which milk and dairy products (Parisi et al., 2016; Basanisi et al., 2017). Thus, the role of food-producing animals and food products as vectors of $S$. aureus, MRSA included, in the food chain is of great concern. Pseudomonads are involved in the spoilage of many chilled food of animal origin; in milk and dairy products they are responsible for off-odours, off-flavours, decreased cheese yields, discolorations (Arslan et al., 2011). The species Ps. fluorescens is the main causative agent of blue discoloration of fresh cheeses (Martin et al., 2011) and of mozzarella cheese, in particular (Cenci Goga et al., 2014). In this study, Ps. fluorescens isolated from blue mozzarella cheese were studied. Concerning the pigment production, the use of YEGB, which is also available on the market for bacterial cultivation and maintenance purposes, could be a cheap and simple tool for food industry to monitor the presence of pigmenting pseudomonads, either in the production environments or in products, even if the test requires previous isolation of microorganisms.

Antimicrobial activity of EOs has been documented since the mid-twentieth century (Burt, 2004). Many studies have focused on the antimicrobial properties of different EOs against $S$. aureus, comprising MRSA. Particularly thyme and cinnamon and their major constituents often showed interesting results. However the tested bacterial strains were generally reference strains or clinical isolates (Smith-Palmer et al., 1998; Jia et al., 2011; Boskovic et al., 2015; ReyesJurado et al., 2016). Regarding our study, we evaluated the antimicrobial activity of four EOs (bergamot, cinnamon, manuka and thyme) against wild food strains, isolated from milk and dairy products, of Ps. fluorescens and biofilm-producing $S$. aureus, MRSA included, in addition to reference strains. As to manuka, the antimicrobial properties of its honey have been studied

Table 2. Minimal inhibitory concentration of four essential oils against the tested Pseudomonas fluorescens and Staphylococcus aureus.

\begin{tabular}{|c|c|c|c|c|}
\hline & & & $\mathrm{v} / \mathrm{v})$ & \\
\hline & $\mathrm{Cb}$ & $\mathrm{Cz}$ & Ls & $\mathrm{Tv}$ \\
\hline Ps. fl. PS59 & 3.125 & 0.195 & 3.125 & 0.195 \\
\hline Ps. fl. PS60 & 3.125 & 0.195 & 3.125 & 0.195 \\
\hline Ps. fl. PS63 & 3.125 & 0.391 & 3.125 & 0.195 \\
\hline Ps. fl. PS70 & 3.125 & 0.391 & 3.125 & 0.098 \\
\hline Ps. fl. PS71 & 3.125 & 0.391 & 3.125 & 0.195 \\
\hline Ps. fl. PS231 & 3.125 & 0.391 & 3.125 & 0.195 \\
\hline Ps. fl. PS249 & 3.125 & 0.391 & 3.125 & 0.098 \\
\hline Ps. fl. PS251 & 3.125 & 0.391 & 3.125 & 0.098 \\
\hline Ps. fl. PS282 & 3.125 & 0.391 & 3.125 & 0.195 \\
\hline Ps. fl. ATCC 13525 & 3.125 & 0.391 & 3.125 & 0.098 \\
\hline S.a. PAl & 0.781 & 0.049 & 0.012 & 0.024 \\
\hline Sa. PA2 & 1.563 & 0.049 & 0.012 & 0.024 \\
\hline S.a. PIl & 1.563 & 0.049 & 0.012 & 0.024 \\
\hline S.a. ATCC 35556 & 1.563 & 0.098 & 0.024 & 0.024 \\
\hline S.a. ATCC 12600 & 1.563 & 0.049 & 0.012 & 0.024 \\
\hline
\end{tabular}

Eos, essential oils; Cb, Citrus bergamia; Cz, Cinnamomum zeylanicum; Ls, Leptospermum scoparium; Tv, Thymus vulgaris; S.a., Staphylococcus aureus; Ps.fl., Pseudomonas fluorescens. Results are the mode of three independent trials. 
more than that of its EO (Mandal and Mandal, 2011). Generally, in our study we observed that the EO resulted to be much more active on Gram positive bacteria than on Gram negative, as reported also by Maddock-Jennings et al. (2005); in addition, van Klink et al. (2005), documented in particular the remarkable antimicrobial effect of EO major compounds, $\beta$ trichetones, on MRSA. Moreover, we recently found in another study (Fratini et $a l ., 2017)$ very interesting MIC results for manuka EO, comparable to those obtained with our biofilm-forming $S$. aureus, on different $S$. aureus strains from milk (methicillin-susceptible and not biofilm-producing strains). Data about bergamot EO are scanty: a study performed by Fisher and Phillips (2006) using different Gram positive and negative microorganisms as target bacteria showed that $\mathrm{Cb}$ EO was more effective against the Gram-positive bacteria. $S$. aureus was less affected, if compared with Listeria monocytogenes and Bacillus cereus. More recently, Marotta et al. (2016) evidenced a strain-dependent activity of bergamot $\mathrm{EO}$ on L. monocytogenes from food samples. In our research $\mathrm{Cb}$ EO was effective on $S$. aureus only at high concentrations.

Overall, it is known that Gram-negative bacteria are more resistant to the EOs effect, due to the hydrophilic lipopolysaccharides contained in the outer membrane, which create a barrier against EOs hydrophobic antimicrobial compounds (Hyldgaard et al., 2012). Generally, Ps. fluorescens sensitivity to EOs has been poorly investigated. Outtara et al. (1997) reported that cinnamon EO was more active than thyme EO on $P S$ fluorescens isolated from beef, whereas we found a higher activity of Tv EO. Also, we found MIC values of about $1-2 \mu \mathrm{L} / \mathrm{mL}$ and 2-4 $\mu \mathrm{L} / \mathrm{mL}$, for Tv and $\mathrm{Cz}$ EOs respectively, against Ps. fluorescens ATCC 13525. Similarly, Mith et al. (2014) found that PS. fluorescens ATCC 13525 was more resistant than other Gram positive and Gram-negative reference strains to the different EOs tested and among which cinnamon and thyme, with MICs ranging from 1 to 1.5 $\mu \mathrm{L} / \mathrm{mL}$.

\section{Conclusions}

The need for natural alternatives to synthetic chemicals is increasing in food industry, as well as the consumers request for natural products. Our study provides interesting data about EOs sensitivity of food-related wild microorganisms, which may be useful in implementing new solutions for sanification of food facilities surfaces and/or for direct use in food and packaging, particularly in the dairy production chain.

\section{References}

Adams RP, 1995. An identification of essential oil components by gas chromatography-mass spectroscopy. Allured Publishing Co, Carol Stream, IL, USA.

Arslan S, Eyi A, Özdemir F, 2011. Spoilage potential and antimicrobial resistance of Pseudomonas spp. isolated from cheeses. J Dairy Sci 94:5851-6.

Basanisi MG, La Bella G, Nobili G, Franconieri I, La Salandra G, 2017. Genotyping of methicillin-resistant Staphylococcus aureus (MRSA) isolated from milk and dairy products in South Italy. Food Microbiol 62:141-6.

Boskovic M, Zdravkovic N, Ivanovic J, Janjic J, Djordjevic J, Starcevic M, Baltic MZ, 2015. Antimicrobial activity of thyme (Thymus vulgaris) and oregano (Origanum vulgare) essential oils against some food-borne microorganisms. Procedia Food Sci 5:18-21.

Burt S, 2004. Essential oils: their antimicrobial properties and potential applications in food-a review. Int $\mathrm{J}$ Food Microbiol 94:223-53.

Cenci Goga BT, Karama M, Sechi P, Iulietto MF, Novelli S, Mattei S, 2014. Evolution under different storage conditions of anomalous blue coloration of Mozzarella cheese intentionally contaminated with a pigment-producing strain of Pseudomonas fluorescens. J Dairy Sci 97:6708-18.

De Buyser ML, Dufour B, Maire M, Lafarge V, 2001. Implication of milk and milk products in food-borne diseases in France and in different industrialised countries. Int J Food Microbiol 67:1-17.

Di Ciccio P, Normanno G, Pedonese F, Nuvoloni R, Parisi A, Santagata G, Caruso M, Zanardi E, Ghidini S, Ianieri A, 2016. Investigation of biofilm formation and its association with biofilm associated factors of food-related methicillin-resistant Staphylococcus aureus strains. LXX Atti S.I.S.Vet. 2612.

Di Ciccio P, Vergara A, Festino AR, Paludi D, Zanardi E, Ghidini S, Ianieri A, 2015. Biofilm formation by Staphylococcus aureus on food contact surfaces: Relationship with temperature and cell surface hydrophobicity. Food Control 50:930-36.

Fisher K, Phillips CA, 2006. The effect of lemon, orange and bergamot essential oils and their components on the survival of Campylobacter jejuni, Escherichia coli O157, Listeria monocytogenes, Bacillus cereus and Staphylococcus aureus in vitro and in food systems. J Appl Microbiol 101:1232-40.

Fratini F, Casella S, Leonardi M, Pisseri F, Ebani VV, Pistelli L, Pistelli L, 2014. Antibacterial activity of essential oils, their blends and mixtures of their main constituents against some strains supporting livestock mastitis. Fitoterapia 96:1-7.

Fratini F, Mancini S, Turchi B, Friscia E, Pistelli L, Giusti G, Cerri D, 2017. A novel interpretation of the Fractional Inhibitory Concentration index: the case Origanum vulgare L. and Leptospermum scoparium J. R. et G. Forst essential oils against Staphylococcus aureus strains. Microbiol Res 195:11-7.

Hyldgaard M, Mygind T, Meyer RL, 2012. Essential oils in food preservation: mode of action, synergies, and interactions with food matrix components. Front Microbiol 3:12.

Jia P, Xue YJ, Duan XJ, Shao SH, 2011. Effect of cinnamaldehyde on biofilm formation and sarA expression by methicillin-resistant Staphylococcus aureus. Lett Appl Microbiol 53:409-16.

Lucera A, Costa C, Conte A, Del Nobile M, 2012. Food applications of natural antimicrobial compounds. Front Microbiol 3:287.

Maddock-Jennings W, Wilkinson JM, Shillington D, Cavanagh H, 2005. A fresh look at manuka and kanuka essential oils from New Zealand. Int J Aromather 15:141-6.

Mandal MD, Mandal S, 2011. Honey: its medicinal property and antibacterial activity. Asian Pac J Trop Biomed 1:154-60.

Marotta SM, Giarratana F, Parco A, Neri D, Ziino G, Giuffrida A, Panebianco A, 2016. Evaluation of the antibacterial activity of bergamot essential oils on different Listeria monocytogenes strains. Ital J Food Saf 5:210-3.

Martin NH, Murphy SC, Ralyea RD, Wiedmann M, Boor KJ, 2011. When cheese gets the blues: Pseudomonas fluorescens as the causative agent of cheese spoilage. J Dairy Sci 94:317683.

Mith H, Duré R, Delcenserie V, Zhiri A, Daube G, Clinquart A, 2014. Antimicrobial activities of commercial essential oils and their components against food-borne pathogens and food spoilage bacteria. Food Sci Nutr 2:403- 
16.

Outtara B, Simard RE, Holley RA, Piette GJP, Bégin A, 1997. Antibacterial activity of selected fatty acids and essential oils against six meat spoilage organisms. Int J Food Microbiol 37:155-62.

Parisi A, Caruso M, Normanno G, La Torre L, Sottili R, Miccolupo A, Fraccalvieri R, Santagada G, 2016. Prevalence, antimicrobial susceptibility and molecular typing of Methicillin-Resistant Staphylococcus aureus (MRSA) in bulk tank milk from southern Italy. Food Microbiol 58:36-42.

Pedonese F, D'Ascenzi C, Torracca B, Zingoni C, Turchi B, Fratini F, Nuvoloni R, 2014. Staphylococcus aureus growth and enterotoxin production in Italian caciotta cheese. Turk J Vet Anim Sci 38:318-24.
Reyes-Jurado F, López-Malo A, Palou E, 2016. Antimicrobial activity of individual and combined essential oils against foodborne pathogenic bacteria. J Food Prot 79:309-15.

Scarpellini M, Franzetti L, Galli A, 2004. Development of PCR assay to identify Pseudomonas fluorescens and its biotype. FEMS Microbiol Lett 236:257-60.

Smith-Palmer A, Stewart J, Fyfe L, 1998. Antimicrobial properties of plant essential oils and essences against five important food-borne pathogens. Lett Appl Microbiol 26:118-22.

Stenhagen E, Abrahamsson S, McLafferty FW, 1974. Registry of mass spectral data. Wiley \& Sons, New York, NY, USA.

Valeriano C, Coutinho de Oliveira TL, Malfitano de Carvalho S, das Graças
Cardoso M, Alves E, Hilsdorf Piccoli R, 2012. The sanitizing action of essential oil-based solutions against Salmonella enterica serotype Enteritidis S64 biofilm formation on AISI 304 stainless steel. Food Control 25:673-7.

van Klink JW, Larsen L, Perry NB, Weavers

RT, Cook GM, Bremer PJ, MacKenzie AD, Kirikae D, 2005. Triketones active against antibiotic-resistant bacteria: synthesis, structure-activity relationships, and mode of action. Bioorg Med Chem 13:6651-62.

Wiegand I, Hilpert K, Hancock RE, 2008. Agar and broth dilution methods to determine the minimal inhibitory concentration (MIC) of antimicrobial substances. Nat Protoc 3:163-75. 